TESIS

Para optar por el título de Doctor en Medicina

\title{
TITULO
}

\section{PAMIDRONATO ENDOVENOSO VS. ALENDRONATO ORAL EN EL TRATAMIENTO DE LA OSTEOPOROSIS ESTABLECIDA}

Autor

Eliseo Hernán Ferrari

Medico MP 17623

\section{DIRECTORES}

Profesor Dr. Ricardo Cerda

Profesor Dr. Alfredo Vitale 


\section{ÍNDICE}

Definición

Epidemiología

3

Manifestaciones Clínicas

5

Formas secundarias

Detección

Indicaciones de tratamiento

Tratamiento

Calcio y vitamina $D$

Bifosfonatos

\section{Eficacia}

Seguridad

Fundamento del plan de tesis

Hipótesis

Objetivos

Material y Métodos

Resultados

Efectos adversos

Discusión 


\section{INTRODUCCION}

\section{Definición}

Se define a la Osteoporosis como una enfermedad metabólica de Hueso caracterizada por una disminución de la masa ósea con deterioro de la microarquitectura del tejido óseo, que da lugar a fragilidad con el consiguiente incremento del riesgo de fracturas ${ }^{1,2,3,7}$.

Según las recomendaciones de la Organización Mundial de la Salud (OMS) 1,2,3 se considera que hay Osteopenia cuando la masa ósea se encuentra entre 1 y 2,5 desvíos estándar por debajo de la media del adulto joven, reservando él término Osteoporosis para una masa ósea por debajo de 2,5 desvíos estándar y Osteoporosis severa cuando se ha complicado con fracturas.

\section{Epidemiología de la osteoporosis}

La osteoporosis tiene una prevalencia elevada en la población. Adoptando la definición previamente mencionada de la OMS, alrededor de $15 \%$ de las mujeres jóvenes tienen osteopenia y $0,6 \%$ osteoporosis ${ }^{1}$. Según fuentes de la OMS 13 a 18 $\%$ de las mujeres en Estados Unidos tienen osteoporosis y 37 a 50 \% osteopenia 7 .

La prevalencia de osteoporosis aumenta con la edad. Entre los 60 a 70 años solo 1 de cada 9 mujeres tiene una masa ósea dentro de límites normales y al menos una de cada tres tiene osteoporosis.

Se calcula que en Estados Unidos alrededor de 10 millones de personas tienen osteoporosis y 33 millones baja masa ósea ${ }^{8}$. En Europa para el año 2010, la prevalencia de osteoporosis se calculaba en $22 \%$ entre las mujeres y $7 \%$ entre los varones mayores de 65 años. Esta cifra se eleva a $47 \%$ de las mujeres y $16 \%$ de los varones por encima de los 80 años ${ }^{4}$.

En Argentina sucede algo similar, se calcula que una de cada 4 mujeres mayores a 50 años tiene osteoporosis y 2 de cada 4 tienen osteopenia ${ }^{3,24}$.

La presencia de osteoporosis ocasiona aumento de riesgo de fracturas, aunque se discute hasta qué punto la medición de la densidad mineral ósea, que es el parámetro para la definición que adopto la OMS, podría predecir el riesgo de fracturas futuras 12. Parecería ser que menos de $1 / 3$ de las fracturas son atribuidas exclusivamente a la baja masa ósea, con lo cual otros elementos menos mensurables tiene que ver en su desarrollo ${ }^{12}$ 
Las fracturas más relacionadas con la presencia de osteoporosis son las de cadera, columna vertebral y radio distal, aunque también tienen que ver con ella las de costillas y pelvis ${ }^{7}$.

En Estados Unidos se producen unos 2 millones de fracturas por año atribuidas a osteoporosis 8. Aproximadamente 300.000 son fracturas de cadera, 547.000 fracturas de columna vertebral y 135.000 de pelvis ${ }^{8}$

La incidencia de fracturas de cadera es de 2 por mil a los 65 años y aumenta a 30 por mil luego de los 85 años ${ }^{1}$. En Argentina la incidencia de fracturas de cadera es de 298 /cien mil en mujeres y 118/cien mil en varones ${ }^{24}$. El riesgo a lo largo de la vida de una mujer de desarrollar una fractura de cadera es de 15 a $17 \%$ y de $6 \%$ para los varones ${ }^{9}$

El pronóstico es variable, y las fracturas de cadera son las más severas. Tienen una mortalidad de 12 a $30 \%$ al cabo del primer año 18,24,76. Entre un 30 a $50 \%$ de los pacientes recuperan la capacidad funcional y un $30 \%$ de los pacientes se vuelven dependientes para todas sus actividades ${ }^{18,24}$. Se suma a ello que la población más expuesta son los gerontes. El $90 \%$ de las fracturas de cadera ocurren en personas mayores de 70 años de edad ${ }^{18}$. En Argentina la edad promedio de las fracturas de cadera es de 82 años en las mujeres y 79 años en los varones ${ }^{24}$.

Los costos económicos una fractura de cadera son muy elevados y se calculan en alrededor de 10 billones de dólares en Estados Unidos ${ }^{76}$.

Otra de las fracturas fuertemente relacionadas a presencia de Osteoporosis son las de columna vertebral. Su prevalencia varía según los distintos trabajos. Se puede calcular el riesgo global de desarrollar una fractura de Columna vertebral a lo largo de la vida en un 15 a $16 \%$ de las mujeres ${ }^{1,7,9}$ y un $5 \%$ de los varones ${ }^{1,7,9}$. La posibilidad de desarrollar una fractura de columna de alrededor de 0,4 \% por año para las mujeres de 65 a 69 años, y de 1,9 \% por año para las mujeres de más de 75 años 1 .

La mayor parte de las fracturas vertebrales son hallazgos radiológicos. Podrían encontrarse evidencias radiológicas de fracturas vertebrales en la mitad de las mujeres a los 85 años ${ }^{10,15}$.

En Argentina la prevalencia de fracturas vertebrales como hallazgo radiológico es de $16 \%$ entre mujeres mayores de 50 años, pero llega a $25 \%$ entre las mujeres mayores de 80 años ${ }^{24}$. 
El riesgo de desarrollar fracturas de Radio distal también está relacionado con la presencia de Osteoporosis. Se calcula que la posibilidad de desarrollar una fractura de Radio a lo largo de la vida de la mujer es de alrededor de un 17 \% 1, 7 .

La aparición de las diferentes fracturas suele tener una relación cronológica con la edad y la menopausia. Las fracturas de radio suelen presentarse a una edad media de 55 años en las mujeres, aproximadamente diez años después se observan las de columna vertebral y las de cadera suelen aparecer otros diez años más tarde, tal como se observa en la figura 1.

Figura 1 tomada de Campion J. Am Fam Phys 2003; 67:1521).

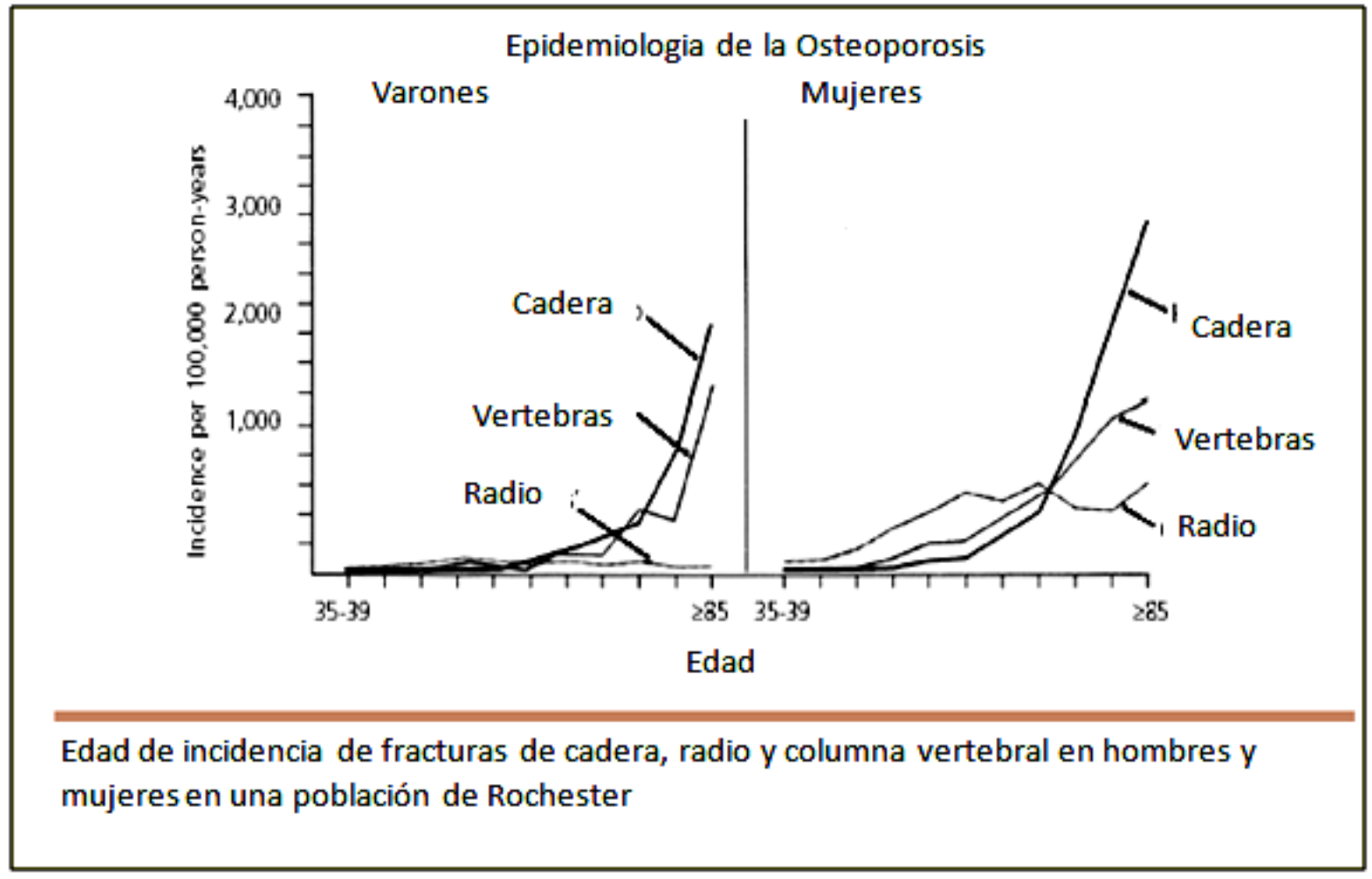

\section{Manifestaciones clínicas}

La osteoporosis no suele dar síntomas en la mayoría de los casos. Aunque en ocasiones algunos pacientes pueden presentar dolor lumbar o dorsal como motivo de consulta consecuencia de fracturas previas que pasaron desapercibidas en su periodo agudo. En estos casos es difícil discernir cual es el origen del dolor, dado que a menudo se trata de pacientes ancianos con múltiples patologías, donde el dolor puede ser ocasionado por Artrosis lumbar, Osteoartritis interfascetaria, Canal medular estrecho y Cifosis o Escoliosis, entre otras causas ${ }^{19}$. 
Las fracturas vertebrales en la mayor parte de los casos no ocasionan síntomas y suelen ser hallazgos radiológicos ${ }^{10,15}$. Se calcula que solo un $30 \%$ de las fracturas vertebrales se pueden presentar como dolor agudo y un $10 \%$ pueden derivar en una internación por la intensidad el dolor. Las fracturas pueden adoptar diferentes formas. Se define como Acuñamiento cuando disminuye la altura anterior de la vértebra respecto a la posterior en más de un $20 \%$. Otro tipo de fractura es la central, con desarrollo de vértebras de aspecto bicóncavo, también denominadas vértebras de pescado. En este caso se expresa como hundimiento en la parte medial de los platillos vertebrales tomando una variación de $20 \%$ como límite. Finalmente un tercer tipo de fractura es el aplastamiento, cuando la vértebra se colapsa en su totalidad (figura 2).

Figura 2. Tipos de fracturas vertebrales

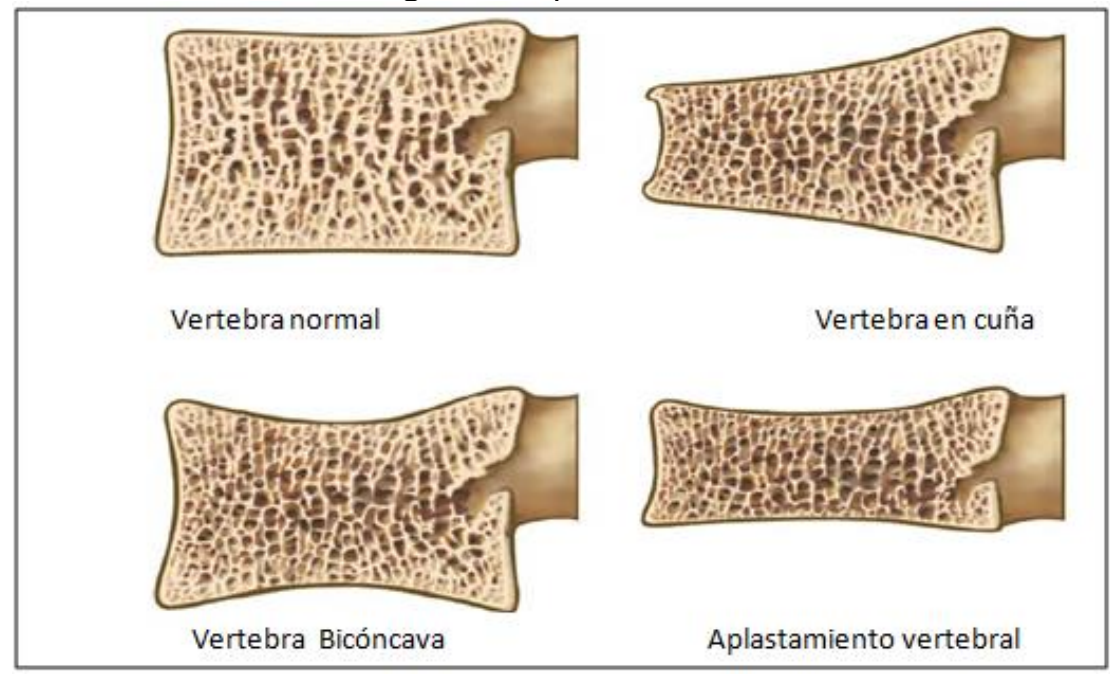

Otras veces los pacientes se presentan con deformidades vertebrales. Es común que se observe cifosis dorsal, que si es muy marcada obliga a hiperextender el cuello ocasionando dolor cervical y fatiga muscular. Se ha dado en denominar "giba de viuda" a esta deformidad típica de las mujeres ancianas con osteoporosis.

Otro síntoma frecuente es la perdida de altura, aunque en este último caso otras situaciones como la pérdida de altura de los discos intervertebrales tiene mucho que ver ${ }^{20}$. Cuando se ha producido una fractura vertebral el riesgo de progresión y desarrollo de una nueva fractura es muy alto, llegando al $20 \%$ dentro del año ${ }^{14}$.

Se suele clasificar a la osteoporosis en formas primarias y secundarias. Las formas primarias representan alrededor del $75 \%$ de los casos de osteoporosis. Se 
destacan como entidades más comunes las denominadas Osteoporosis Posmenopáusica y Senil que representan la mayor parte de los casos.

La osteoporosis pos menopáusica suele producirse como consecuencia de la caída de los niveles de estrógenos. Los estrógenos ejercen sobre el hueso efectos directos e indirectos que resultan en una inhibición de la resorción ósea. Como consecuencia hay un incremento de la actividad osteoclástica que crea profundas cavidades resortivas que no alcanzan a ser compensadas por la actividad osteoblástica, lo que constituye una osteoporosis de alto recambio óseo ${ }^{21}$. La mayor pérdida de masa ósea se produce durante los 5 a 10 años posteriores a la última menstruación. En esta etapa de la vida en condiciones normales, se puede llegar a perder entre 1 a $3 \%$ de masa ósea anual. En las mujeres con osteoporosis pos menopáusica la pérdida ósea es mayor en el hueso trabecular. Esto se debe a la mayor actividad metabólica del hueso esponjoso que lo torna más vulnerable al aumento del recambio óseo. Por esta razón los huesos con elevado contenido esponjoso tales como vértebras, pelvis y radio distal se hallan más comprometidos.

En la osteoporosis post menopáusica cuando se desarrollan fracturas, asientan en columna vertebral y radio distal, por ser huesos de predominio trabecular y metabólicamente más activos ${ }^{12,13}$.

La osteoporosis postmenopáusica tiene un recambio óseo acelerado, donde se forman profundas cavidades resortivas por aumento de la actividad osteoclástica, que no pueden ser compensadas con la actividad osteoblástica ${ }^{22,23}$.

Otra de las formas comunes es la osteoporosis senil. No es fácil establecer un límite neto de la anterior, pero en general se produce en individuos de mayor edad, por encima de 75 años. Es difícil establecer un punto de corte y un paciente evidentemente pasará por una u otra forma según el momento de la vida que consulte, por la cual muchos cuestionan la practicidad de esta división.

En el desarrollo de la osteoporosis en el anciano intervienen varios mecanismos. Por una parte hay una menor ingesta de calcio, además se agrega una deficiencia en la absorción del calcio intestinal ${ }^{24,25}$. Los ancianos tienen menores niveles de vitamina $D$, por menor exposición solar y como consecuencia menor producción cutánea. También hay una menor capacidad de la piel en sintetizar vitamina $\mathrm{D}$, dependiente de la edad y reducción de la actividad de la 1 alfa hidroxilasa del aparato yuxtaglomerular renal, con menor producción de 1-25 hidroxivitamina D que es la forma activa. 
En la osteoporosis senil el recambio (turnover) óseo se encuentra reducido con una menor actividad de los osteoblastos.

Los pacientes con osteoporosis senil, suelen tener afección del hueso cortical y trabecular. Tienen tendencia a desarrollar fracturas de columna vertebral y fémur ${ }^{24}$.

\section{Formas secundarias de osteoporosis}

Una de las formas más comunes es la osteoporosis esteroide, que se desarrolla en 30 a $50 \%$ de los pacientes que ingieren corticoides con fines terapéuticos, similar en su comportamiento al síndrome de Cushing 27 .

Los mecanismos de acción son pobremente comprendidos, pero entran en juego varios factores, como menor absorción de calcio intestinal, asociado a mayor excreción renal de calcio que se hace evidente a la semana del inicio de la terapia. Es secundario a un efecto directo de los corticoides sobre el transporte tubular del calcio.

Todo esto facilita un balance cálcico negativo que ocasiona un hiperparatiroidismo secundario. Esto genera un aumento de la actividad osteoclástica y mayor reabsorción ósea ${ }^{28,29}$.

La actividad de los osteoblastos está reducida en los pacientes que reciben esteroides, colaborando con el balance negativo ${ }^{28}$ La actividad de los osteocitos también se ve afectada, con apoptosis de los mismos inducida por los esteroides y compromiso de vascularización del hueso.

La osteoporosis esteroidea tiene relación con la dosis aunque es difícil definir un umbral por debajo de la cual no se produzca compromiso óseo. En pacientes con artritis reumatoide dosis promedio de $5 \mathrm{mg}$ diarios ocasionan una pérdida de masa ósea de $2 \%$ por año en la columna lumbar, que puede aminorarse con aporte de vitamina $\mathrm{D}$ y calcio ${ }^{30}$.

Los corticoides afectan principalmente el hueso trabecular con desarrollo de fracturas de columna vertebral, costillas y pelvis dado su contenido óseo mayormente representado por hueso trabecular 29

Existen otras formas secundarias de osteoporosis de causa endocrinológica como ser Hipogonadismo, Hiperprolactinemia, Hipertiroidismo y Anorexia nerviosa (Tabla 1). Se agregan otras causas secundarias como ser mala absorción, drogas y enfermedades reumatológicas. 
Tabla 1. Clasificación de la osteoporosis

\begin{tabular}{|ll|}
\hline PRIMARIAS & \\
INVOLUTIVAS \\
Osteoporosis pos menopáusica \\
Osteoporosis senil & \\
Idiopática juvenil & \\
SECUNDARIAS & \\
ENDOCRINAS & NUTRICIONAL \\
Osteoporosis Esteroidea & Alcoholismo \\
Hipertiroidismo & Operaciones gástricas \\
Hiperprolactinemia & Mala absorción \\
Hiperparatiroidismo & Déficit de Vitamina D \\
Anorexia Nerviosa & ENFERMEDADES CRONICAS \\
Hemocromatosis & Amiloidosis \\
DROGAS & Espondilitis \\
Agonistas Gn RH & EPOC \\
Corticoides & Hepatopatías \\
Ciclosporina & AR \\
Fenobarbital & LES \\
Fenitoina & Mieloma \\
Heparina & \\
\hline
\end{tabular}

\section{Detección de Osteoporosis}

Es materia de debate, cual es la mejor aproximación para el manejo de los pacientes con sospecha de osteoporosis. La adopción de la medición densitométrica, adoptando los umbrales establecidos por la OMS es una de las posibilidades. Sin embargo, esta aproximación ha sido cuestionada, dado que menos de un tercio de las fracturas son consecuencia directa de la baja masa ósea ${ }^{12}$.

Muchas sociedades científicas recomiendan indicar densitometría a toda mujer luego de los 65 años 3,31,34. En esto coinciden tanto la "Fundación Nacional de Osteoporosis (NOF) de los Estados Unidos, la "Sociedad Americana de Menopausia" y la "US Preventive Task Force americana" 31,34.

En nuestro medio el Consenso Argentino de Osteoporosis coincide con esta misma recomendación ${ }^{3,34}$.

En las mujeres menores de 65 años existen diferentes posturas. En general la mayor parte de las sociedades científicas coinciden sobre la necesidad de realizar densitometría por debajo de esta edad, aunque no hay acuerdo sobre qué criterio de selección emplear. 
Las normas de la "US Preventive Task Force" americana sugieren que la detección para mujeres menores de 65 años, podría ser de utilidad hasta los 60 años, siempre y cuando tengan factores de riesgo de osteoporosis ${ }^{32}$. La Task Force no recomienda detección rutinaria a mujeres menores de 60 años por que no la consideran una estrategia costo efectiva ${ }^{32}$.

El Consenso Argentino recomienda una densitometría, como detección en la mujer pos menopáusica menor de 65 años que tenga por lo menos uno o más factores de riesgo de osteoporosis o enfermedades asociadas a baja masa ósea. Entre los factores de riesgo tenemos 3,34 :

- Historia personal de fracturas previas

- Antecedente de fractura en familiares de primer grado

- Enfermedades asociadas a baja masa ósea

- Menopausia precoz < 40 años o quirúrgica < 45 años

- Bajo peso (IMC < $\left.20 \mathrm{~kg} / \mathrm{m}^{2}\right)$

- Tabaquismo

- Mieloma

- Enfermedades hematológicas

- Inmovilización.

- Carencia de estrógenos en la pre menopausia

- Trasplante de órganos

- Ingesta de corticoides u otras drogas asociadas a osteoporosis

- Amenorrea primaria

- Bajo consumo de calcio

- Inmovilización prolongada

A esta lista se le agregan por otra parte las enfermedades asociadas a baja masa ósea a las cuales se recomienda estudiar, entre ellas tenemos:

- Trastornos de conducta alimenticia

- Osteomalacia

- Hiperparatiroidismo

- Hipertiroidismo

- Hipogonadismo 
- Síndrome de Cushing

- Hiperprolactinemia con trastornos del ciclo menstrual

- Insuficiencia renal crónica

- Diabetes

- Alcoholismo

- Hipercalciuria

- Hepatopatías

- Mala absorción

- Gastrectomía

- Artropatías inflamatorias

El Consenso Argentino, al igual que la guías de la "National Osteoporosis Foundation“ (NOF) de los Estados Unidos no ponen límites a la edad de los pacientes para indicar una densitometría como técnica de detección. En ambos casos jerarquizan los factores de riesgo para la toma de decisiones.

En esta línea de trabajo, se han desarrollado varios sistemas de puntuación, que agrupando los diferentes factores de riesgo tratan de establecer un sistema de detección que permita predecir riesgo de fracturas y necesidad de realizar densitometría. Entre ellos tenemos el sistema SCORE, el sistema ORAI, las guías de la NOF y el sistema propuesto por la OMS, denominado FRAX.

El sistema de puntuación ORAI otorga 15 puntos si la persona tiene una edad mayor a 75 años, 9 puntos por edad de 65 a 74 años y 5 puntos por edad de 55 a 64 años. Se otorgan además 9 puntos si el peso corporal es menor a $60 \mathrm{Kg}$ y 3 si el peso se halla entre 60 y $69 \mathrm{~kg}$. Si la paciente no recibió terapia hormonal de reemplazo le agrega 2 puntos adicionales. Se recomienda estudiar con este sistema a todas las mujeres con un puntaje mayor a 15. La sensibilidad es de $99 \%$ y la especificidad de $36 \% 35$.

La densitometría, si bien muchos la objetan, ha demostrado ser buen predictor del riesgo de fracturas, aunque no el único. El estudio Rotterdam ${ }^{33}$ en un seguimiento de 4731 mujeres y 3075 varones estudiados por densitometría seguidos por 7 años, demostró que por cada desvió estándar de reducción de la densidad mineral ósea aumentaba la tasa de fracturas no vertebrales $40 \%$ (RR 1,4 IC95\% de 1,2 a 1,6) y un $80 \%$ las fracturas vertebrales (RR 1,8 IC95\% de 1,3 a 2,4 ). Un resultado similar 
mostró el estudio MROS en varones y SOF ${ }^{36}$ en mujeres, aunque la densitometría de cadera con presencia de osteoporosis fue más predictiva de riesgo de fractura en varones que en mujeres.

De acuerdo a las recomendaciones actuales, se asume que en parte la fragilidad ósea puede predecirse a través de la realización de una densitometría o a través de sistemas de cálculo ${ }^{12}$. La OMS recientemente diseño un sistema de cálculo para predecir el riesgo de fracturas en los próximos 10 años, aplicando variables sencillas del interrogatorio.

Este sistema de puntuación, denominado FRAX, se basa en datos obtenidos de un seguimiento de 46.340 personas, provenientes de 9 cohortes, subsiguientemente testeados en otra cohorte de 230.340 personas ${ }^{16}$. Sin embargo, en un estudio comparativo el sistema FRAX no parece superar al esquema tradicional basado en la densitometría para decidir el tratamiento ${ }^{37}$.

\section{Indicaciones de tratamiento}

Las indicaciones de tratamiento son materia de debate y hay discrepancias entre las principales sociedades científicas ${ }^{3}$. A continuación enumeramos las indicaciones de tratamiento y sus controversias:

1.- Pacientes con $\mathrm{t}$ score menor a 2,5 de acuerdo a las recomendaciones de todas las sociedades. Sin embargo, la "NOF" recomienda inicio de tratamiento con T score menor a 2, al igual que el Consenso Argentino en mujeres pos menopáusicas

2.- Mujeres con T Score entre 1 y 2,5 , pero un riesgo de fracturas mayor a $3 \%$ a 10 años en cadera y $20 \%$ a 10 años en columna con un sistema de puntuación validado como el sistema FRAX propuesto por la OMS ${ }^{11}$. *

3.- Mujeres pre menopáusicas y varones con osteoporosis **

4.-Pacientes que van a recibir corticoides por más de 3 meses a dosis mayores a $5 \mathrm{mg} /$ día con un $\mathrm{T}$ score $<1$ ***

5.- Mujeres pos menopáusicas con una fractura previa por osteoporosis

6.- Algunos recomienda iniciar tratamiento con un $Z$ score, que es la densidad mineral ósea comparada con las mujeres de su misma edad, menor a 1,5 en individuos mayores de 80 años

* El sistema de cálculo FRAX, diseñado por la OMS, permite predecir el riesgo de fractura de una población determinada en los próximos 10 años. Algunas sociedades científicas como la "NOF" sugieren tratamiento de todos los pacientes con 
un T Score entre 1 y 2,5 D.S. con un riesgo de fracturas de cadera mayor a $3 \% 0$ mayor a $20 \%$ en otras regiones, independientemente de los valores obtenidos en la densitometría ${ }^{12,16}$.

Las críticas a esta recomendación nos muestran que si adoptáramos este sistema, implicaría que $72 \%$ de las mujeres americanas mayores de 65 años deberían recibir tratamiento, cifra que se elevaría a $93 \%$ de las mayores de 75 años ${ }^{12,37}$.

${ }^{*}$ Se incluyen entre las indicaciones de tratamiento a las mujeres pre menopáusicas y los varones con osteoporosis. Asimismo, las mujeres pos menopáusicas con una fractura previa por osteoporosis.

${ }^{* * *}$ Se recomienda tratar a los pacientes que van a recibir corticoides por más de 3 meses a dosis mayores a $5 \mathrm{mg} /$ día cuyo T score se halle entre $<1$ a 1,5 desvíos estándar, umbral que varía según diferentes sociedades científicas ${ }^{27}$.

\section{Tratamiento de la osteoporosis}

El tratamiento de la osteoporosis consta de medidas no farmacológicas y farmacológicas. Se debe asegurar una adecuada nutrición con aporte de calcio. A partir de los 50 años se debería asegurar un aporte de calcio de la dieta de alrededor de $1200 \mathrm{mg}$ diarios. Además el ingreso de proteínas que debería ser de al menos 1 $\mathrm{g} / \mathrm{kg} /$ día $^{3}$.

Se debe promover actividad física, adecuada a las condiciones del paciente. El ejercicio físico permite fortalecer los músculos y reducir en forma secundaria las caídas. La actividad debe ser planificada de acuerdo a las capacidades del paciente, pero puede aumentar la masa ósea entre un 1 a $2 \%$ 3,12,17. Son recomendados las caminatas o natación evitando cargas sobre las articulaciones y huesos ${ }^{43}$.

Una adecuada exposición solar de al menos 10 a 15 minutos 2 veces diarias fuera de los horarios pico, permite síntesis de vitamina D en la piel. En los ancianos es recomendable que se haga examen de agudeza visual en forma periódica y cuidar el manejo de su hogar evitando todo tipo de obstáculos caseros como cables, alfombras y patines, es decir que todo aquello que facilite las caídas. Muchos psicofármacos de uso frecuente en los ancianos ocasionan problemas de equilibrio, así que se debe ser cuidadoso con su uso ${ }^{3,24}$. El tabaquismo influye en forma negativa sobre la masa ósea, por tal razón es aconsejable dejar de fumar ${ }^{3,24}$. Los protectores de cadera han demostrado efectividad en algunos estudios reduciendo el riesgo de fracturas de cadera alrededor de un $50 \%$. 
Se debe asegurar un aporte adecuado de calcio y vitamina D. A ello se suman las drogas específicas para el tratamiento que pueden clasificarse por su mecanismo de acción en antirresortivas, estimulantes de la formación ósea y drogas de mecanismo complejo ${ }^{34}$ (Tabla 2$)^{6}$.

Tabla 2. Clasificación de las drogas específicas para la osteoporosis

\begin{tabular}{|l|l|l|}
\hline \multicolumn{1}{|c|}{ Antirresortivas } & Estimulantes formación & Mecanismo poco claro \\
\hline Bifosfonatos & Teriparatide & Estroncio \\
Calcitonina & Fluoruro de sodio & \\
Terapia hormonal & & \\
Raloxifeno & & \\
Tibolona & & \\
Denozumab & & \\
\hline
\end{tabular}

\section{Calcio y vitamina D}

Las Guías argentinas de Osteoporosis recomiendan aporte de calcio no menor a 1000 a 1500 mg/día en la mujer luego de la menopausia como base de todo tratamiento ${ }^{3}$. De manera similar otras Guías como las de la "NOF" sugieren como parte del tratamiento calcio $1200 \mathrm{mg}$ y vitamina D al menos 800 u/día ${ }^{44}$.

Sin embargo han surgido en los últimos años cuestionamientos a esta recomendación a partir de recientes estudios ${ }^{44}$. Hay pocos trabajos con aporte exclusivo de calcio sin vitamina $\mathrm{D}^{45}$.

La vitamina $D$ en dosis mayores a 800 u día ha demostrado reducir el riesgo de fracturas en meta análisis ${ }^{46,47}$. También mejora la fuerza muscular y reduce el riesgo de caídas ${ }^{48}$. Los efectos parecen ser mayores en pacientes ancianos institucionalizados, que tal vez tengan carencia de vitamina $D^{43}$

Un reciente meta análisis cuestiona el aporte de suplementos de calcio en los pacientes. En el análisis de 26 estudios con un total de 58.573 pacientes el aporte de calcio y vitamina $D$ redujo el riesgo de fracturas totales $11 \%(0,89$ IC95\% 0,81 a 0,96), pero en términos absolutos el beneficio fue menor con un NNT de 489. También redujo el riesgo de fracturas vertebrales $14 \%(0,86$ IC95\% 0,74 a 1.00). No mostró efectos en la fracturas de cadera ${ }^{49}$. 
Sobre la seguridad de los suplementos de calcio existen algunas dudas. Se ha planteado que podrían ocasionar un aumento del riesgo cardiovascular.

Un meta análisis mostró un incremento del riesgo de infarto de $31 \%$ en los pacientes que recibieron suplementos de calcio ${ }^{50,51}$. Esto implicaría según los autores de esta revisión ${ }^{51}, 6$ nuevos casos de infarto adicionales por cada 1000 pacientes tratados. Se generaron fuertes críticas a este trabajo, por adjudicación inconsistente de eventos, resultados marginales y sobre todo por exclusión de estudios relevantes como el WHI, precisamente este estudio que incluyó 36.282 mujeres, no demostró aumento del riesgo cardiovascular ${ }^{43}$. Precisamente un meta análisis, con inclusión del estudio WHI, no mostró aumento del riesgo de infarto (RR 1,04 IC95\% de 0,92 a $1,18)^{52}$.

El aporte de calcio puede ocasionar aumento del riesgo de litiasis, aunque es discreto y dosis dependiente ${ }^{43}$.

En términos relativos el aumento de riesgo es de $17 \%$, pero en términos absolutos es mínimo con un NNH de $261^{43}$. Producen constipación en 10 a $20 \%$ de los pacientes ${ }^{43}$.

No hay evidencias de que el aporte dietético sea diferente a los suplementos de calcio respecto a los potenciales beneficios ${ }^{43}$.

Más allá de todas las controversias el aporte de calcio y vitamina D está recomendado en todos los tratamientos de osteoporosis ${ }^{3,34}$

En este estudio todas las pacientes recibieron aporte de calcio en dosis de 500 mg dos veces por día como carbonato de calcio y vitamina D2 5000 u por semana.

\section{Bifosfonatos}

Los Bifosfonatos son compuestos caracterizados por dos enlaces entre átomos de carbono y fósforo. Los Bifosfonatos son las drogas de elección en el tratamiento de la osteoporosis ${ }^{3,34}$

Por esta estructura particular se denomina a la molécula P.C.P. Unida a la estructura básica P.C.P, se encuentran dos cadenas laterales llamadas R1 y R2 que permiten la introducción de numerosas substituciones que resultan en la síntesis de una variante de análogos de diferentes propiedades farmacológicas ${ }^{8}$. La cadena R1 determina la afinidad de la unión al hueso y la cadena $\mathrm{R} 2$ la potencia antiresortiva ${ }^{8}$ (Figura 3) 


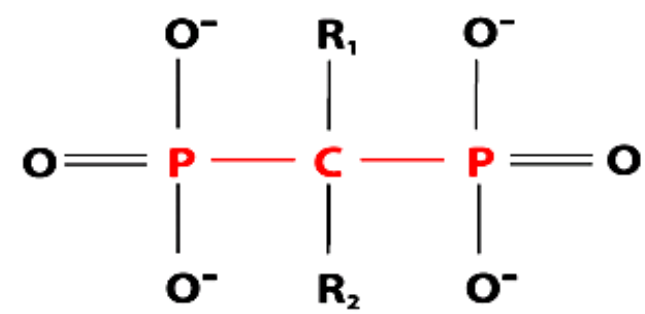

Figura 3. Estructura básica de los bifosfonatos

Los Bifosfonatos se fijan en los cristales de hidroxiapatita e inhiben su disolución ${ }^{8,37}$. Son atrapados en forma irreversible en la matriz mineral, fijándose en la profundidad del hueso trabecular. Inhiben la enzima farnesil pirofosfato sintetasa de los osteoclastos, que participa en el ciclo del mevalonato $8,37,38$, e interfiere con la isoprenilación de las moléculas de guaniltrifosfato del borde rugoso del osteoclasto, afectando su capacidad de unirse a la superficie ósea ${ }^{8,37,38}$. Los Bifosfonatos además inducen apoptosis de osteoclastos 8,38 .

Su capacidad de unirse a las superficies óseas por largo tiempo les confiere un efecto residual que permite esquemas semanales, mensuales y anuales ${ }^{38}$.

\section{Eficacia}

En la tabla 3 se presentan los principales Bifosfonatos disponibles y sus características farmacológicas. Entre los más evaluados tenemos Alendronato, Risedronato, Ibandronato y Zoledronato (tabla 4).

Alendronato produce significativa ganancia de masa ósea. En el estudio FIT I 53 , en pacientes con osteoporosis establecida y fracturas previas al cabo de 3 años se logró un incrementó la masa ósea $6,2 \%$ en columna vertebral y 4,1 \% en cuello de fémur. 
Tabla 3. Principales Bifosfonatos disponibles

\begin{tabular}{|c|c|c|c|}
\hline DROGA & Cadena R1 & Cadena R2 & Potencia \\
\hline Etidronato * & $-\mathrm{OH}$ & $-\mathrm{CH} 3$ & 1 \\
\hline Clodronato * & $-\mathrm{Cl}$ & $-\mathrm{Cl}$ & 10 \\
\hline Pamidronato & $-\mathrm{OH}$ & $-\mathrm{CH} 2-\mathrm{CH} 2-\mathrm{NH} 2$ & 100 \\
\hline Alendronato & $-\mathrm{OH}$ & $-\mathrm{CH} 2-\mathrm{CH} 2-\mathrm{CH} 2-\mathrm{NH} 2$ & 1000 \\
\hline Risedronato & $-\mathrm{OH}$ & $-\mathrm{CH}_{2}-\stackrel{=\mathrm{N}}{=}$ & 5000 \\
\hline lbandronato & $-\mathrm{OH}$ & $\begin{array}{c}-\mathrm{CH}_{2}-\mathrm{CH}_{2}-\mathrm{NH}_{2}-\mathrm{CH}_{3} \\
\mathrm{C}_{5} \mathrm{H}_{11}\end{array}$ & 10.000 \\
\hline Zolendronato & $-\mathrm{OH}$ & $-\mathrm{CH}_{2}-\mathrm{N}=\mathrm{N}$ & 20.000 \\
\hline
\end{tabular}

Además Alendronato obtuvo una reducción del riesgo de nuevas fracturas vertebrales y no vertebrales, aunque el efecto fue más evidente en las fracturas vertebrales, la mayor parte de las cuales son asintomáticas. Los efectos sobre las fracturas de cadera fueron menos significativos, expresado en términos relativos se logró una reducción de $51 \%$, pero en términos absolutos fue solo $1 \%$. La eficacia de Alendronato en pacientes sin fracturas previas, fue evaluada en el marco del estudio FIT II, donde los pacientes fueron seleccionados solo por su baja masa ósea ${ }^{54}$. En este caso la reducción de fracturas vertebrales en términos relativos fue $44 \%$, pero en términos absolutos fue de $1,7 \%$.

En análisis de subgrupos el efecto beneficioso se vio limitado a los pacientes con $\mathrm{T}$ score menor a 2,5 desvíos estándar ${ }^{54}$. Por ende no tenemos evidencia de reducción en tasa de fracturas en pacientes con osteopenia ${ }^{38}$.

Risedronato reduce fracturas vertebrales y no vertebrales en pacientes con baja masa ósea y fracturas previas ${ }^{55}$.

Sus efectos sobre las fracturas de cadera son modestos, en términos absolutos demostró una reducción entre 1,3 a 1,6 \% 55,56

Ibandronato en dosis de 2,5 $\mathrm{mg}$ diarios redujo nuevas fracturas vertebrales morfométricas en un $52 \%{ }^{57}$. En las fracturas no vertebrales no mostró diferencias.

Los Bifosfonatos han sido ensayados en prevención de la osteoporosis pos menopáusica demostrando aumento de masa ósea, pero sin diferencias en la tasa de fracturas 58 . 
Los Bifosfonatos se absorben muy poco por vía oral, alcanzando una biodisponibilidad de 0,7 \%. Los alimentos reducen su absorción. La vida media en circulación es muy breve alrededor de 20 a 120 minutos y tienen una rápida redistribución con fijación a hueso. Se eliminan por riñón sin transformase ${ }^{37,38}$.

Para mejorar la adherencia y aprovechando sus características particulares farmacocinéticas se han ensayado las dosis semanales de $70 \mathrm{mg}$ de Alendronato, que se mostraron igualmente efectivas que las dosis diarias respecto a la ganancia de masa ósea, pero no se evaluó la tasa de fracturas con esta posología 59.

De la misma manera las dosis semanales de $35 \mathrm{mg}$ de Risedronato se mostraron eficaces comparadas a dosis diarias en la ganancia de la masa ósea ${ }^{60}$.

Ibandronato en dosis mensual de 150 mg también se mostró eficaz en la evolución de la masa ósea comparado a dosis diarias ${ }^{61}$

En una revisión sistemática de los principales trabajos publicados con Bifosfonatos, que incluyó 15 estudios, aunque muchos de ellos de pobre calidad metodológica. Se llegó a la conclusión que los Bifosfonatos reducen riesgo de fracturas vertebrales alrededor de un $34 \%$ (RR 0,66 con un IC95\% de 0,50 a 0,89). Aunque sus resultados como suelen expresarse en reducción de riesgo relativo se ven magnificados ${ }^{16}$.

Si analizamos su eficacia en reducción de riesgo absoluto, los efectos son más modestos, con una diferencia de 3,1 \% en el grupo placebo vs. 1,9\% en el grupo tratamiento, es decir una diferencia de $1,2 \%{ }^{16}$. Por otra parte la mayor parte de las fracturas de columna vertebral son asintomáticas, es decir hallazgos morfométricos en estudios radiológicos.

Los beneficios en reducción de fracturas no vertebrales, incluidas las de cadera que son las más relevantes por la repercusión clínica, son mucho más modestos ${ }^{16}$. La eficacia de los Bifosfonatos es mayor en pacientes con fracturas previas. 
Tabla 4. Principales ensayos clínicos con Bifosfonatos

\begin{tabular}{|c|c|c|c|c|}
\hline $\begin{array}{l}\text { OTSG } \\
\text { N Engl J. } \\
\text { Med. 1995; } \\
\text { 333:22/1437-1442. }\end{array}$ & N: 994 & T score $<2,5$ & $\begin{array}{l}\text { Fracturas } \\
\text { vertebrales } \\
\text { RR } 0,52 \text { IC } 0,28 \text { a } \\
0,92\end{array}$ & $\begin{array}{l}\text { No vertebrales } \\
\text { N-S. }\end{array}$ \\
\hline $\begin{array}{l}\text { FIT I } \\
\text { Edad } 45 \text { a } 80 \text { años }\end{array}$ & 2027 & $\begin{array}{l}\text { T Score }<2 \text { mas } \\
\text { fracturas }\end{array}$ & $\begin{array}{l}\text { Vertebrales RR } \\
0,53(0,41 \text { a } 0,68)\end{array}$ & $\begin{array}{l}\text { Fracturas cadera } \\
\text { RR } 0,49 \text { IC } 0,23 \text { a } \\
0,9\end{array}$ \\
\hline $\begin{array}{l}\text { FIT II } \\
\text { Edad } 55 \text { a } 81 \text { años }\end{array}$ & 4432 & $\begin{array}{l}\text { T score }<0,68 \\
\mathrm{gr} / \mathrm{cm} 2 \text { cadera }= \\
<1,6\end{array}$ & $\begin{array}{l}\text { Fracturas clínicas } \\
\text { (RR } 0,86 \text { IC } 0,73- \\
1,01 \text { ). } \\
\text { Fracturas cadera } \\
\text { (RR } 0,79 \text { IC } 0,43- \\
1,44)\end{array}$ & $\begin{array}{l}\text { T score }>2,5 \\
\text { Fracturas cadera } \\
\text { RR } 0,44 \text { (IC } 0,18- \\
0,97 \text { ) } \\
\text { NNT de } 81 \text {. } \\
\text { score }<2,5 \text { no hubo } \\
\text { (RR } 1,8) .\end{array}$ \\
\hline VERT & 2458 & $\begin{array}{l}\text { Mujeres fracturas } \\
\text { vertebrales } \\
\text { DMO }<2<0,83\end{array}$ & $\begin{array}{l}\text { Fracturas } \\
\text { vertebrales RR } 0,59 \\
(0,43-0,82)\end{array}$ & $\begin{array}{l}\text { Fracturas no } \\
\text { vertebrales fue RR } \\
0.61 \text { (IC } 0.39-0,94)\end{array}$ \\
\hline HIP & 9931 & $\begin{array}{l}5545 \mathrm{~T}<40<3 \text { con } \\
\text { factores de riesgo } \\
3886 \text { edad }>80 \text { con } \\
\text { factores de riesgo }\end{array}$ & & $\begin{array}{l}\text { G 1: Fracturas } \\
\text { cadera } \\
\text { RR } 0,6(0,4 \text { a } 0,9) \\
\text { G 2: N. S. } 0,8 \text { IC } \\
0,6-, 2\end{array}$ \\
\hline
\end{tabular}

En una revisión de la base de datos COCRHANE se observó que en pacientes con fracturas previas el NNT, en fracturas vertebrales fue 16 y en fracturas no vertebrales fue de $100^{62}$. En pacientes sin fracturas previas el NNT fue 50 en columna vertebral pero no fue significativo en fracturas no vertebrales.

Es tema de controversia la duración del tratamiento con Bifosfonatos. La mayor parte de los estudios tuvieron una duración de 3 años. En una extensión a 5 años del estudio FIT, denominada estudio FLEX se observó que había ganancia de masa ósea en la columna vertebral y se mantenía en la cadera.

Los pacientes que abandonaron el tratamiento perdieron masa ósea en columna, aunque de manera leve.

Se obtuvo con la prolongación del tratamiento una reducción de las fracturas vertebrales, pero no hubo diferencias en las fracturas no vertebrales ${ }^{63}$. Luego se hicieron prolongaciones más allá de los 5 años de los estudios originales con Alendronato (estudio FLEX), Zoledronato (estudio HORIZON-MN) y Risedronato 
(VERT-MN). Estas extensiones tuvieron una extensión variable de 6 a 10 años y la FDA evaluó los resultados ${ }^{64}$.

La prolongación más allá de 5 años dio resultados variables. En el estudio HORIZON se redujeron las fracturas morfométricas pero no hubo diferencia en fracturas clínicas, aunque el estudio se suspendió a los 6 años.

En el estudio FLEX, se obtuvo ganancia de masa ósea en columna de $5 \%$ en 5 años vs. 1,5\% en el grupo placebo. En contraposición se observó una reducción de la masa ósea de $1 \%$ en cadera total en el grupo Alendronato vs. Una caída de 3,3\% en el grupo placebo. No hubo diferencias en el riesgo de fracturas totales y no vertebrales. Se observó una reducción en el riesgo de fracturas vertebrales clínicas 70,71

Los resultados analizados por la FDA con Alendronato fueron discordantes. No hubo diferencias en el riesgo de fracturas. Se desarrollaron nuevas fracturas en 17,7 $\%$ del grupo Alendronato vs. 16,9 \% del grupo control.

Similares resultados se pueden observar en otros trabajos de seguimiento. De esta manera se genera la duda si la extensión del tratamiento más allá de los 5 años ofrece alguna ventaja ${ }^{61}$.

\section{Seguridad}

La intolerancia digestiva es uno de los efectos más comunes de los Bifosfonatos. Los pacientes refieren dolor abdominal, náuseas y vómitos en 2 a $10 \%$, los cuales son más comunes con Clodronato, Pamidronato y Tiludronato por vía oral $37,38,65$. Se puede desarrollar esofagitis, lo que parece ser consecuencia de la ingesta del medicamento en horas de la mañana con escasa cantidad de líquido ${ }^{65,66}$. Se considera la presencia de esofagitis severa y acalasia como una contraindicación absoluta para cualquier tratamiento con Bifosfonatos ${ }^{66}$.

En un estudio se mencionó un aumento de riesgo de cáncer de esófago con Bifosfonatos ${ }^{67}$. Sin embargo, un reciente estudio de casos y controles más extenso no parece confirmar este hallazgo 68

Los Bifosfonatos pueden producir efectos adversos musculo-esqueléticos como dolor óseo, fiebre y fatiga. Un síndrome de tipo gripal se puede observar sobre todo con el uso endovenoso. Suele aparecer a los 3 días y perdura entre 7 a 14 días después de la infusión. Hasta $20 \%$ de los pacientes que reciben un Bifosfonato endovenoso lo pueden desarrollar ${ }^{8,65}$. No suele repetirse con las sucesivas infusiones. 
Se pueden desarrollar artralgias y mialgias con Alendronato oral ${ }^{66}$. Las infusiones endovenosas pueden ocasionar hipocalcemia, sobre todo si la velocidad del goteo es muy rápida 65 .

En pocos casos se ha desarrollado insuficiencia renal aguda con uso de Clodronato y Etidronato. En el estudio HORIZON se desarrolló aumento de la creatinina mayor a $0,5 \mathrm{mg} / \mathrm{dl}$ en $1,2 \%$ de los pacientes tratados con Zoledronato endovenoso vs. 0,4 \% del grupo control. En todos los casos hubo recuperación espontánea y no se detectaron diferencias en la función renal al cabo de 3 años de duración del estudio ${ }^{65,66}$.

En el estudio HORIZON también se documentó mayor riesgo de fibrilación auricular (FA), 1,3 vs. $0,4 \%$ en el grupo placebo.

Nuevos estudios se han publicado luego con Alendronato demostrando un aumento discreto del riesgo de FA, aunque hace falta mayor evidencia ${ }^{66}$.

También se han descripto casos de síndrome de ojo rojo por uveítis. Recientemente se han reportado casos de necrosis aséptica en la mandíbula, aunque se han visto con altas dosis de Bifosfonatos endovenosos en pacientes oncológicos, o en enfermedad de Paget ${ }^{37,38}$. Los pacientes forman ulceraciones por las cuales se drena el hueso expuesto, estas úlceras persisten al menos 8 semanas ${ }^{37,38,66}$.

Existen dudas sobre la posibilidad de desarrollo de trastornos de la mineralización ósea y alteraciones en la calidad del hueso ${ }^{38,72}$. Recientemente se han reportado fracturas subtrocantereas en pacientes tratadas con Bifosfonatos ${ }^{72}$. Estas fracturas aparecen ante traumatismo mínimo, se asocian a engrosamiento cortical y pueden ser fracturas incompletas ${ }^{72}$. Se desarrollarían en 4 a 7 casos por cada 10 mil pacientes por año con un $\mathrm{NNH}$ de $2000{ }^{38}$. El riesgo aumenta con el tiempo de tratamiento y comenzaría a partir de 2 años y se incrementa a los 5 años ${ }^{38}$. 


\section{PLAN DE TESIS}

\section{FUNDAMENTO DEL PLAN DE TESIS}

Los Bifosfonatos son las drogas más eficaces y evaluadas en el tratamiento de la osteoporosis, constituyéndose en la primera línea de tratamiento ${ }^{38,39}$. Incrementan la densidad mineral ósea y reducen riesgo de fracturas vertebrales y no vertebrales. Sin embargo una limitante es su pobre absorción oral y la frecuente intolerancia digestiva. Por esta razón la adherencia ha sido uno de los problemas más comunes en el tratamiento con Bifosfonatos. Los pacientes suspenden el tratamiento en más de la mitad de los casos dentro del año ${ }^{39}$.

La particular farmacocinética de los Bifosfonatos permite dosis semanales y mensuales por vía oral, pues se fijan por largo tiempo en los cristales de hidroxiapatita. Aun con todas las alternativas mencionadas la intolerancia digestiva persiste. Por esta razón y aprovechando su farmacocinética particular se ha ensayado el empleo de Bifosfonatos endovenosos con la finalidad de lograr una mayor adherencia y permitir ampliar las indicaciones a pacientes con intolerancia digestiva.

Al inicio de este plan de tesis las opciones de tratamiento eran escasas.

El Pamidronato se había ensayado previamente en el tratamiento de osteoporosis en estudios de diseño abierto ${ }^{40}$, donde se compararon diferentes dosis por vía endovenosa en pacientes con osteoporosis e intolerancia a otros Bifosfonatos por vía oral. En ese marco mostró la misma eficacia que Alendronato en el tratamiento de la osteoporosis ${ }^{41}$

El uso de Pamidronato en osteoporosis es una indicación no avalada por agencias reguladoras internacionales como la FDA de los Estados Unidos y la EMEA europea, es decir que constituye un "Off Label". El Consenso Argentino de Osteoporosis recomienda Pamidronato endovenoso como una opción ante intolerancia a Bifosfonatos por vía oral ${ }^{3}$.

En los últimos años han aparecido otras opciones de uso endovenoso como el Zoledronato ${ }^{61}$, cuyo costo sin embargo limita su uso. También se encuentra disponible el Ibandronato endovenoso ${ }^{62}$, aunque con menores niveles de evidencia sobre su eficacia, pues no ha demostrado reducir el riesgo de fracturas no vertebrales. 


\section{HIPÓTESIS}

La hipótesis de la presente tesis, es que Pamidronato endovenoso sería al menos igual de efectivo que Alendronato por vía oral en el tratamiento de las pacientes con osteoporosis establecida, convirtiéndose de esa manera en una opción de tratamiento cuando existe intolerancia a los Bifosfonatos por vía oral. 


\section{OBJETIVOS}

\section{OBJETIVO PRIMARIO}

El objetivo primario de este estudio fue evaluar la eficacia del Pamidronato endovenoso en pacientes con osteoporosis establecida e intolerancia digestiva a Bifosfonatos orales para mejorar la densidad mineral ósea en la columna vertebral y la cadera.

\section{OBJETIVO SECUNDARIO}

El objetivo secundario fue

a) Evaluar la tasa de fracturas vertebrales y no vertebrales.

b) Evaluar la seguridad de ambas estrategias terapéuticas en términos de efectos adversos reportados para los Bifosfonatos en general y para Pamidronato endovenoso en particular. 


\section{MATERIAL Y MÉTODOS}

Diseño: Estudio de cohorte prospectiva, llevado a cabo en el consultorio externo del Instituto del Diagnóstico de la ciudad de La Plata desde el año 1999 hasta el año 2011, durante un lapso de 12 años.

Criterios de inclusión: Pacientes de sexo femenino, adultas mayores de 45 años, con menopausia establecida, con diagnóstico de osteoporosis de acuerdo a los criterios sugeridos por la OMS (densidad mineral ósea menor a 2,5 desvíos estándar (DS) debajo de la media de las mujeres jóvenes normales utilizadas como comparador). Las pacientes del grupo Pamidronato se seleccionaron por desarrollo de intolerancia a Alendronato oral, por desarrollo de esofagitis definida clínicamente y por endoscopía

Criterios de exclusión: pacientes con Insuficiencia renal crónica con clearence menor a $35 \mathrm{ml} /$ minuto, alergia a Bifosfonatos, carencia de vitamina D no corregida, definida por síntomas clínicos de osteomalacia o niveles menores de $20 \mathrm{ng} / \mathrm{ml}$ de vitamina D sérica. Esofagitis (para el grupo Alendronato), hiperparatiroidismo y enfermedades neoplásicas al momento del diagnóstico,

Estrategia de muestreo: Muestreo de conveniencia. Se incluyeron todas las pacientes consecutivas en seguimiento ambulatorio por osteoporosis durante el período de estudio. Las pacientes fueron reclutadas entre el año 1999 y el año 2004. El estudio culminó en el año 2009, al terminar los periodos de tratamiento pautados en cada grupo de estudio.

Se establecieron dos grupos de tratamiento: un grupo que recibió Pamidronato intravenoso (30 mg cada 3 meses). Este grupo fue reclutado por desarrollo de intolerancia digestiva previa a Alendronato oral. Otro grupo recibió Alendronato oral, en dosis de $70 \mathrm{mg}$ por semana, dado la buena tolerancia digestiva al mismo.

Cointervenciones: todos los pacientes recibieron un aporte de calcio de al menos 1000 mg/día y vitamina $D$ en dosis de 5000 u por semana 
Analítica de laboratorio: Se hicieron análisis al inicio de tratamiento, luego entre los 3 y 6 meses y finalmente cada año. Se compararon las mismas variables al final del tratamiento instituido.

Se analizaron las condiciones generales y las comorbilidades de los pacientes en forma global y en cada grupo al inicio y al final del tratamiento.

El laboratorio general y los valores del metabolismo fosfocálcico se analizaron al inicio de tratamiento y su culminación en el global de pacientes y en cada grupo de tratamiento.

La duración planeada del tratamiento fue al menos 3 años, de acuerdo a la mayor parte de los trabajos publicados de Bifosfonatos según las guías terapéuticas vigentes al momento del inicio del plan de tesis.

Se tomaron los valores de densitometría, con equipo de Densitometría tipo LUNAR. Los punto de medición fueron columna lumbar, promedio en L2 -L4 y cuello del fémur, al inicio de tratamiento y luego cada 1 o 2 años. Se tomaron los valores de la densitometría al inicio y al final del tratamiento para el cálculo de la evolución de la masa ósea con cada rama de tratamiento.

En el caso de las variables continuas el procesamiento estadístico se realizó con prueba de T o análisis de varianza según correspondiera. Se aplicó test de Fisher o de chi cuadrado para las variables nominales.

Se analizó la presencia de nuevas fracturas reportadas por las pacientes o como hallazgo radiológico al final de tratamiento. Para tal fin se hicieron radiografías de columna dorsal de perfil al inicio y al final del tratamiento. Se adoptó como definición de nuevas fracturas vertebrales la presencia de una reducción de la altura anterior de la vértebra más de $4 \mathrm{~mm}$ como se ha hecho en otros estudios de referencia 49

Las comorbilidades de las pacientes, previo al inicio del estudio se analizaron primero en el grupo total y luego, separadas para el grupo Alendronato y Pamidronato. No se encontraron diferencias significativas entre ambos grupos de tratamiento.

Los datos de laboratorio general antes de iniciar el tratamiento se analizaron en el total de la muestra y luego por separado en cada grupo de tratamiento. 


\section{RESULTADOS}

Se reclutaron 43 pacientes, 30 en el grupo Alendronato y 13 en el grupo Pamidronato. Al momento del reclutamiento 12 pacientes tenían fracturas secundarias a osteoporosis, 6 fracturas en cada grupo.

En la tabla 5 se muestran los datos basales del grupo total y de los grupos tratados con Alendronato y Pamidronato. Como se aprecia no hubo diferencias significativas en edad, IMC, hematocrito, eritrosedimentación, leucocitos, creatininemia, colesterolemia, trigliceridemia, calcemia, fosforemia, fosfatasa alcalina, densitometría ósea de la columna lumbar y del cuello femoral.. La glucemia basal fue más alta en el grupo Pamidronato y el HDL-c más bajo en el grupo Pamidronato.

Tabla 5. Datos basales de los participantes del estudio

\begin{tabular}{lccccccc}
\hline & Total, media & DS & Alendronato & DS & Pamidronato & DS & P \\
\hline Edad, años & 64,09 & 9,10 & 63,13 & 8,86 & 66,31 & 9,61 & 0,30 \\
IMC, Kg/m² & 23,21 & 4,23 & 23,21 & 2,64 & 23,52 & 6,78 & 0,83 \\
Hto, \% & 39 & 4 & 40 & 3,8 & 38 & 38 & 0,07 \\
ERS, mm & 18,98 & 20,44 & 16,13 & 14,41 & 25,54 & 29,89 & 0,17 \\
Leucocitos, n\%/mm & 6404 & 1562 & 6246 & 1156 & 6769 & 1644 & 0,33 \\
Glucemia, mg/dL & 86 & 10 & 83 & 11 & 91 & 8 & 0,03 \\
Creatinina, mg/dL & 0,95 & 0,21 & 0,93 & 0,21 & 1,01 & 0,20 & 0,26 \\
Colesterol, mg/dL & 209 & 38,02 & 209 & 35,34 & 208 & 45,17 & 0,93 \\
HDL-C, mg/dL & 53 & 12 & 57 & 13 & 47 & 8 & 0,02 \\
TG, mg/dL & 112 & 41 & 108 & 42 & 123 & 38 & 0,27 \\
Calcio, mg/dL & 9,3 & 0,68 & 9,3 & 0,76 & 9,32 & 0,45 & 0,94 \\
Fósforo, mg/dL & 3,5 & 0,55 & 3,5 & 0,53 & 3,91 & 0,45 & 0,09 \\
FAL, U/L & 171 & 70 & 59 & 61 & 199 & 82 & 0,08 \\
DMO col. lumbar, & & & & & & & \\
g/cm² & 0,82 & 0,08 & 0,82 & 0,08 & 0,84 & 0,08 & 0,73 \\
DMO cuello fémur, & 0,73 & 0,11 & 0,74 & 0,10 & 0,71 & 0,03 & 0,39 \\
\hline g/cm²
\end{tabular}

DS: desvío estándar; IMC: índice de masa corporal; Hto: hematocrito; ERS:

eritrosedimentación; HDL-C: colesterol HDL; TG: triglicéridos; FAL: fosfatasa alcalina; DMO: densidad mineral ósea.

La duración media del tratamiento fue 5 años en el grupo Alendronato y 3,9 años en el grupo Pamidronato.

Los motivos de la suspensión de tratamiento fueron por decisión médica en 28 casos (65\%), 6 por desarrollo de esofagitis (13\%), todos dentro del grupo Alendronato, 
6 casos suspendieron el tratamiento por falta de adherencia (4 en el grupo Alendronato y 2 en el grupo Pamidronato). Tres pacientes fallecieron por sus diferentes comorbilidades, una por desarrollo de leucemia aguda, una por osteomielitis protésica y una por muerte súbita.

Los datos del laboratorio general luego de finalizado el estudio se pueden ver en la tabla 6. Las diferencias entre ambas ramas de tratamiento fueron un mayor valor de ERS, con significación limítrofe, y un número mayor de leucocitos, que ya existía previo al estudio, ambos en el grupo Pamidronato. También, en el grupo Pamidronato, se observó un mayor valor de glucemia y un menor nivel de colesterol HDL, diferencias que ya existían previas al inicio del tratamiento.

Tabla 6. Datos de laboratorio al finalizar el estudio

\begin{tabular}{lccccc}
\hline & Alendronato & DS & Pamidronato & DS & p \\
\hline Hto, \% & 39,3 & 3,14 & 37,9 & 4,78 & 0,27 \\
ERS, mm & 16,4 & 15,74 & 28,85 & 24,11 & 0,05 \\
Leucocitos, $\mathrm{n} / \mathrm{mm}^{3}$ & 5710 & 1836 & 8333 & 338 & 0,03 \\
Glucemia, mg/dL & 85,5 & 11,44 & 93,62 & 10,59 & 0,04 \\
Creatinina, mg/dL & 0,97 & 0,20 & 1,05 & 0,31 & 0,29 \\
Colesterolemia, mg/dL & 215,57 & 34,86 & 194,6 & 38,84 & 0,05 \\
HDL-c, mg/dL & 56,5 & 11,27 & 47,54 & 10,94 & 0,02 \\
TG, mg/dL & 105,13 & 36,07 & 97,38 & 28,91 & 0,50 \\
Calcemia, mg/dL & 9,28 & 0,61 & 9,13 & 0,23 & 0,38 \\
Fósforemia, mg/dL & 3,44 & 0,44 & 3,86 & 0,58 & 0,11 \\
FAL, mg/dL & 148 & 40,01 & 173 & 46,37 & 0,08 \\
\hline
\end{tabular}

Evolución de la masa ósea

La densidad mineral ósea en la columna lumbar, promedio Lumbar 2-4 en el grupo Alendronato al final del tratamiento, fue $0,85 \mathrm{~g} / \mathrm{cm}^{2}$, lo que implica un aumento de $0,03 \mathrm{~g} / \mathrm{cm}^{2}$; expresado en porcentaje representa un incremento de $4,60 \%(p=0,017)$ (tabla 7 , figs. 4 y 5 ). 
Tabla 7. Masa ósea pre y postratamiento con alendronato y pamidronato

\begin{tabular}{clccccccc}
\hline Droga & \multicolumn{1}{c}{ DMO } & $\begin{array}{c}\text { Pre } \\
\text { tratamiento* }\end{array}$ & DS & $\begin{array}{c}\text { Post } \\
\text { tratamiento* }\end{array}$ & DS & $\begin{array}{c}\text { Diferencia } \\
\text { post- pret }^{*}\end{array}$ & $\%$ & P \\
\hline Alendronato & Lumbar 2-4 & 0,82 & 0,07 & 0,85 & 0,08 & 0,03 & 4,60 & 0,017 \\
& Cuello fémur & 0,74 & 0,10 & 0,74 & 0,09 & - & - & 0,78 \\
Pamidronato & Lumbar 2-4 & 0,84 & 0,08 & 0,90 & 0,11 & 0,06 & 7,38 & 0,002 \\
& Cuello fémur & 0,71 & 0,03 & 0,71 & 0,13 & - & - & 0,93 \\
\hline
\end{tabular}

*gramos/cm²; DMO: densidad mineral ósea; DS: desvío estándar

La densidad mineral ósea en el cuello del fémur en el grupo Alendronato al terminar el estudio no tuvo significación estadística (tabla 7).

En el grupo Pamidronato la densidad mineral ósea en la columna lumbar al final del tratamiento fue $0,90 \mathrm{~g} / \mathrm{cm}^{2}$, lo que implica un aumento de $0,06 \mathrm{~g} / \mathrm{cm}^{2}$; expresado en porcentaje representa un incremento de $7,38 \%(p=0,002)$ (tabla 7$)$. Comparados los efectos sobre la columna lumbar entre ambos tratamientos no tuvieron diferencias significativas.

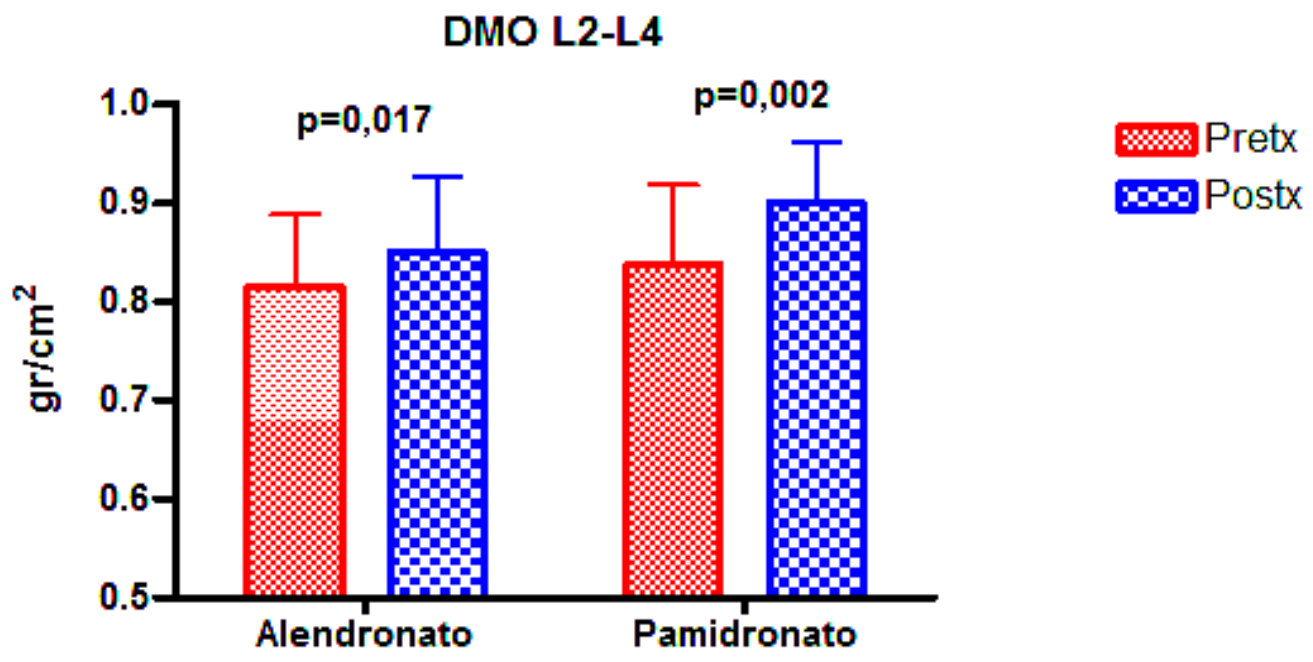

Figura 4: Evolución de la masa ósea. Diferencia entre pre y postratamiento expresada en $\mathrm{g} / \mathrm{cm}^{2}$ 
DMO L2-L4

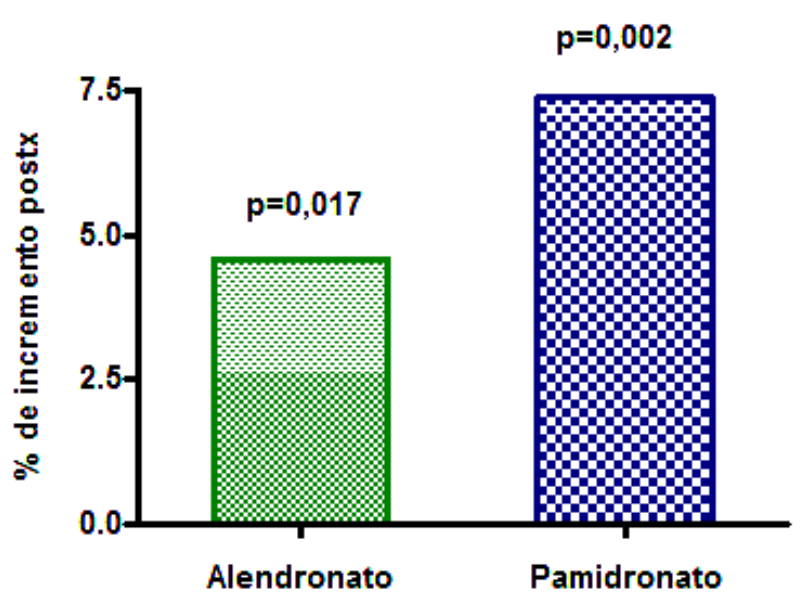

Figura 6. Evolución de la masa ósea. Diferencia entre pre y postratamiento expresada en \% de incremento.

En el grupo Pamidronato la densidad mineral ósea en el cuello del fémur al final del tratamiento no mostró diferencias con significación estadística (tabla 7).

Como podemos observar se obtuvo un significativo aumento de la masa ósea en la columna lumbar en ambos grupos de tratamiento, sin diferencias entre ellos, pero no se lograron beneficios en el cuello del fémur.

\section{Seguridad - efectos adversos}

En los pacientes del grupo Alendronato fue frecuente el desarrollo de intolerancia digestiva, aun habiendo descartado previamente a aquellos con estos antecedentes. Se desarrollaron síntomas de esofagitis en 7 casos del grupo Alendronato (23\%). En 5 casos los síntomas digestivos fueron lo suficientemente severos como para motivar el acortamiento de los plazos de tratamiento.

En todos los casos se realizó una endoscopía digestiva alta para el diagnóstico. En 3 casos la endoscopía fue normal, en 3 se observó esofagitis erosiva y en una de las pacientes se desarrolló una úlcera de esófago. Una de las pacientes tuvo síntomas compatibles con espasmo del esófago durante el tratamiento, su endoscopía mostró esofagitis y los síntomas desaparecieron con el tratamiento con omeprazol y la suspensión del Alendronato. 
En los pacientes del grupo Pamidronato en dos casos luego de la infusión se reportaron mialgias y artralgias sin fiebre. Al prolongar el tiempo de infusión la sintomatología no se repitió.

En el grupo Alendronato tres pacientes desarrollaron nuevas fracturas, todas en la columna vertebral; no se constataron nuevas fracturas en el grupo Pamidronato. Esta diferencia no fue significativa (test de Fisher, $p=0,09$ ). 


\section{DISCUSIÓN}

En este estudio de cohorte prospectiva se comparó en pacientes con osteoporosis. el tratamiento con Pamidronato endovenoso contra un grupo tratado con Alendronato oral.

Al finalizar el estudio se observó un aumento de la masa ósea en la columna lumbar de 4,6\% en el grupo Alendronato y de 7,4\% en el grupo Pamidronato, similar al de otros estudios. Así, en el estudio FIT I ${ }^{53}$ el aumento de densidad mineral ósea en la columna fue $6,2 \%$ y $8,3 \%$ en el estudio FIT II ${ }^{54}$. Con Risedronato la ganancia de masa ósea en la columna fue $5,4 \%$.

Los resultados obtenidos en este estudio mostraron que ambos tratamientos, Alendronato y Pamidronato, fueron efectivos para aumentar la densidad mineral ósea de la columna lumbar.

La mayor ganancia de masa ósea obtenida por Pamidronato, comparado con Alendronato, si bien no alcanzó significación estadística demostró que es una alternativa al menos con la misma eficacia para el tratamiento de la osteoporosis.

En este estudio no se observaron diferencias en la masa ósea de la cadera.

Pero hay que tener prudencia con la interpretación de los resultados. La medición por densitometría tiene un margen de error de 1\% para la columna vertebral y de $1,5 \%$ para el cuello del fémur ${ }^{3}$. Cambios inferiores a $2,77 \%$ en la columna y $4,15 \%$ en el cuello del fémur pueden ser debidos a errores en la precisión del método 3 .

La estabilidad de los valores de la masa ósea entre mediciones seriadas podría considerarse como un criterio de respuesta terapéutica según las recomendaciones del Consenso Argentino de Osteoporosis ${ }^{3}$. En ese mismo sentido solo se interpretaría que una pérdida mayor a $4 \%$, podría ser consecuencia de una mala respuesta a un tratamiento instituido. Por ende el mantenimiento de la masa ósea durante tres años se asocia a reducción en la tasa de fracturas ${ }^{69}$. Por todo esto podríamos asumir que la estabilidad observada en ambos grupos de tratamiento en la evolución de la masa ósea de la cadera se podría interpretar como una respuesta terapéutica favorable.

La duración del tratamiento en el grupo Alendronato fue de 5 años, comparado con 3,92 años en el grupo Pamidronato. Esta diferencia en la duración de los tratamientos fue estadísticamente significativa, aunque no parece haber tenido 
influencia en la respuesta obtenida pues no hubo un mayor aumento de la masa ósea en los pacientes del grupo Alendronato.

Cuando comparamos la duración de este estudio con los principales estudios clínicos con Bifosfonatos encontramos que la mayor parte de ellos, como el FIT I y II ambos con Alendronato ${ }^{53,54}$ y los estudios VERT y HIP con Risedronato ${ }^{55,56}$, tuvieron una duración de 3 años. Por esta razón se asume que la duración de este estudio puede considerarse apropiada a la luz de las recomendaciones actuales ${ }^{37,38}$.

Cuando se inició este plan de tesis no se ponían límites a la duración del tratamiento, asumiendo que la osteoporosis era una enfermedad crónica, con lo cual era usual prolongar los tratamientos mucho más allá de los 5 años ${ }^{37,38}$, aunque no había evidencias firmes sobre la eficacia a largo plazo. La prolongación del tratamiento más allá de los 3 años mostraba ganancia de la masa ósea en la columna lumbar, aunque la misma tendía a reducirse.

La Intolerancia digestiva es uno de los efectos más comunes de los Bifosfonatos. Los pacientes refieren dolor abdominal, náuseas y vómitos en 2 a 10\%, los cuales son más comunes con Clodronato, Pamidronato y Tiludronato por vía oral 37,38,65. Se puede desarrollar esofagitis, la que parece ser consecuencia de la ingesta del medicamento en horas de la mañana con escasa cantidad de líquido ${ }^{65,66}$. La presencia de esofagitis severa y acalasia se considera como una contraindicación absoluta para cualquier tratamiento con Bifosfonatos ${ }^{66}$.

Respecto a la intolerancia digestiva, en este trabajo fue limitada a los pacientes del grupo Alendronato. Se desarrolló esofagitis en la cuarta parte de los pacientes y motivó la suspensión del tratamiento en 4 casos. En todos los casos se hicieron estudios endoscópicos documentando esofagitis en 3 casos y una úlcera de esófago en un caso.

Los Bifosfonatos pueden producir efectos adversos musculoesqueléticos como dolor óseo, fiebre y fatiga. Un síndrome de tipo gripal se puede observar sobre todo con el uso endovenoso ${ }^{8,65}$. En el presente trabajo los efectos adversos musculoesqueléticos en el grupo Pamidronato fueron dolores articulares y musculares leves que no necesitaron la suspensión del tratamiento. Se desarrollaron en 3 pacientes, fueron controlados con paracetamol oral y se atenuaron en las infusiones subsiguientes. 
Los pacientes se mostraron renuentes a la infusión endovenosa en todos los casos. Esta dificultad en la forma de administración del Pamidronato pudo tener influencia en la duración más acotada del tratamiento.

El Pamidronato había sido ensayado previamente en el tratamiento de la osteoporosis en estudios de diseño abierto ${ }^{40}$. Se compararon en ese estudio diferentes dosis por vía endovenosa en pacientes con osteoporosis e intolerancia a otros bifosfonatos por vía oral. El estudio tuvo un número reducido de pacientes, a diez de ellos se les administró $30 \mathrm{mg}$ de Pamidronato EV cada 3 meses y a otros 10 , $30 \mathrm{mg}$ semanales por 4 semanas. No se demostraron diferencias entre ambos esquemas de tratamiento.

En otro estudio ${ }^{41}$ se comparó Alendronato oral en dosis de $10 \mathrm{mg} / \mathrm{día}$ vs. Pamidronato EV en dosis de $60 \mathrm{mg}$ cada 3 meses. Participaron 20 pacientes en cada grupo y el estudio tuvo un año de duración, al cabo del cual se evaluó la densidad mineral ósea en la cadera y la columna vertebral. Se obtuvo una ganancia de masa ósea de $4 \%$ en la columna vertebral en ambos grupos y en el cuello del fémur de 3,3 y $2,9 \%$ respectivamente. Los autores concluyeron que Pamidronato puede ser utilizado exitosamente en los pacientes con intolerancia a los Bifosfonatos por vía oral. La ganancia de masa ósea se parece a la obtenida en nuestro estudio, aunque emplearon una dosis mayor de Pamidronato y la posología de Alendronato fue diaria.

Luego del inicio de la tesis se publicaron 2 nuevos trabajos con Pamidronato.

En un estudio retrospectivo similar al presente realizado en Holanda comparó la eficacia de Alendronato $70 \mathrm{mg}$ por semana vs. Risedronato $35 \mathrm{mg}$ por semana y Pamidronato $60 \mathrm{mg}$ cada 3 meses. Este último solo en pacientes con intolerancia a Bifosfonatos por vía oral. Los pacientes fueron reclutados si habían completado al menos 2 años de tratamiento. La duración media del tratamiento fue finalmente de 4 años. La ganancia de masa ósea en la columna vertebral en el grupo con tratamiento oral fue $8,3 \%$ y en el grupo Pamidronato $6,2 \%$. En la cadera la ganancia de masa ósea fue similar, $1,1 \%$ en el grupo con tratamiento oral y $1,4 \%$ en el grupo Pamidronato. Las diferencias no fueron estadísticamente significativas concluyendo que Pamidronato es una buena alternativa ante intolerancia a los Bifosfonatos por vía oral 74.

Otras opciones de tratamiento endovenoso surgieron luego del plan de tesis, entre ellas Zolendronato e Ibandronato. 
El Estudio HORIZON ${ }^{75}$, tuvo como objetivo evaluar la eficacia del Zolendronato endovenoso anual en el tratamiento de la osteoporosis. Se reclutaron 7665 mujeres posmenopáusicas de 65 a 89 años con un T score $<2,5$ DS en el cuello del fémur o < 1,5 asociado a dos o más fracturas vertebrales previas. Las variables primarias elegidas para analizar fueron nuevas fracturas vertebrales o fracturas de cadera. Las variables secundarias fueron otras fracturas, evolución de la masa ósea y marcadores séricos de osteoporosis. Las pacientes fueron randomizadas a recibir ácido zoledronico $5 \mathrm{mg}$. administrados en 15 minutos o placebo a los 0,12 y 24 meses. Todas recibieron calcio y vitamina D. Al cabo de 3 años se logró una ganancia de masa ósea de $6 \%$ en la columna vertebral y de $5 \%$ en la cadera. Pero lo más importante fue que se redujo $70 \%$ el desarrollo de nuevas fracturas vertebrales (RR 0,30; IC95\% 0,24-0,38). Se redujeron $25 \%$ las fracturas no vertebrales, $33 \%$ todas las fracturas y $77 \%$ las fracturas clínicas. La reducción obtenida en las fracturas de la cadera fue $41 \%$ (RR 0,59; IC95\% 0,42-0,83). Sin embargo los efectos adversos fueron serios. Se desarrolló insuficiencia renal aguda en $1,3 \%$ de los pacientes con Zolendronato vs. $0,4 \%$ del grupo control. Los valores retornaron a la normalidad en 30 días en $85 \%$ de los pacientes y no se desarrolló insuficiencia renal crónica en 3 años de seguimiento. Se produjeron arritmias, sobre todo fibrilación auricular en 1,4\% vs. 0,4\% del grupo control.

También se encuentra disponible Ibandronato endovenoso. En un estudio se compararon diferentes dosis de Ibandronato por vía oral, $2,5 \mathrm{mg} / \mathrm{día}$ vs. $2 \mathrm{mg}$ EV y 3 mg EV cada 3 meses. La variable primaria elegida para analizar fue la elevación de la densidad mineral ósea. Al cabo de 2 años se obtuvo una mayor ganancia de masa ósea con la administración de altas dosis por vía EV comparada con la vía oral ${ }^{76}$. Se cuestiona que Ibandronato no ha demostrado reducir el riesgo de las fracturas no vertebrales que son las más significativas desde el punto de vista estadístico ${ }^{77}$.

El estudio de esta tesis tuvo un número reducido de pacientes en comparación con los previamente mencionados financiados por la industria farmacéutica. Sin embargo, la ganancia de masa ósea obtenida fue significativa y si bien hubo menos fracturas en el grupo Pamidronato, no alcanzó significación estadística probablemente por el escaso número de eventos y pacientes. De todas maneras los estudios precedentes con Pamidronato, también tuvieron un número reducido de pacientes reclutados como se ha analizado. 
El Pamidronato no está aprobado por las agencias reguladoras como la FDA de los Estados Unidos y la EMA europea. Sin embargo el Consenso Argentino de Osteoporosis recomienda Pamidronato endovenoso como una opción ante intolerancia a los Bifosfonatos por vía oral ${ }^{3}$.

Este estudio tuvo varias limitaciones.

La primera de ellas es el tipo de diseño. No fue un estudio randomizado doble ciego, los pacientes del grupo Pamidronato se reclutaron por intolerancia a los Bifosfonatos. Este tipo de diseño fue consecuencia de que los pacientes fueron tomados del mundo real, no en el marco de un ensayo clínico controlado. Esto desde ya que pudo influir en los resultados. Sin embargo hemos visto y comentado estudios de diseño similar en la bibliografía consultada referentes a Pamidronato.

Otra limitación es que la duración del tratamiento no fue uniforme, en el grupo Alendronato fue de 5 años y en el grupo Pamidronato de 3,92 años, esto podría subestimar los efectos del Pamidronato al acortar el lapso de tratamiento. La menor duración del tratamiento del grupo Pamidronato tuvo que ver con una menor adherencia al régimen endovenoso por la incomodidad del manejo que requiere una infusión de, al menos, 4 a 6 horas con una internación breve en sistemas ambulatorios de "hospital de día".

La tercera limitación es que la variable de estudio elegida inicialmente fue la evolución de la densidad mineral ósea en la cadera y la columna vertebral. Desde ya que es más importante, para evaluar la eficacia de una droga en la osteoporosis, elegir como variable la reducción de la tasa de fracturas. En el estudio no se detectaron diferencias significativas en la tasa de fracturas, probablemente por el limitado número de pacientes incluidos. La ganancia de densidad mineral ósea en la columna fue similar con ambos tratamientos, aunque la diferente duración pudo influir subestimando la ganancia obtenida por el Pamidronato. 


\section{CONCLUSIÓN}

La industria farmacéutica a través de su patrocinio permite la financiación de grandes estudios cuando estos son rentables a futuro. Obviamente esta situación no sería posible para Pamidronato con lo cual difícilmente tengamos un estudio de grandes dimensiones sobre esta modalidad de tratamiento.

Pamidronato endovenoso podría ser una alternativa de tratamiento más económica que las otras disponibles por vía endovenosa, como Zolendronato e Ibandronato. La desventaja es que requiere una infusión endovenosa, a pasar en 4 horas, lo que demanda atención de enfermería con costos indirectos que se deben tomar en consideración.

En resumen, Pamidronato podría ser una alternativa de tratamiento en pacientes con intolerancia a los Bifosfonatos aunque harían falta mayores trabajos. 


\section{BIBLIOGRAFÍA}

1. Ross P. Osteoporosis. Arch Int Med 1996; 156:1399-1411.

2. Gass M. Preventing Osteoporosis-Related Fractures: An Overview The American Journal of Medicine (2006) Vol 119 (4A), 3S-11S.

3. Guías para diagnóstico, prevención y tratamiento de la osteoporosis Revista Argentina de Osteología 2007; 6: 27-39.

4. Hernlund E, Svedbom A, Ivergard M, et al. Osteoporosis in the European Union: medical management, epidemiology and economic burden. A report prepared in collaboration with the International Osteoporosis Foundation (IOF) and the European Federation of Pharmaceutical Industry Associations (EFPIA). Arch Osteoporos 2013; 8:136

5. Khosla S. Osteopenia. N Engl J Med. 2007; 356:2293-30

6. Cummings S. Epidemiology of Osteoporotic fractures. Lancet 2002; 359:1761-8.

7. Ettinger M. Aging Bone and Osteoporosis. Arch Int Med 2003; 163:2237.

8. Favus MJ. Bisphosphonates for Osteoporosis. N Engl J Med 2010; 363:2027-35.

9. South J. Osteoporosis evaluation AFP 2001;63:898

10. Sambrock P. Osteoporosis. Lancet 2006; 367:2010-18

11. Järvinen TL, Michaëlsson $\mathrm{K}$, Jokihaara $\mathrm{J}$, et al. Overdiagnosis of bone fragility in the quest to prevent hip fracture BMJ 2015;350:h2088 doi: 10.1136/bmj.h2088 (Published 26 May 2015)

12. Ensrud K. Vertebral fractures. N Engl J Med 2011; 364: 634

13. Papaianou A. Diagnosing and management of vertebral fractures. Am J Med. 2002; 113:220.

14. Nagathanan V. Vertebral Fractures. Arch Int Med 2001; 160:2917.

15. Nelson H. Screening for osteoporosis. Ann Int Med 2010; 153:99

16. Weinstein R. Glucocorticoid induced bone disease. N Engl J Med 2011; 365:62

17. Brunner LC, Eshilian-Oates L. Hip Fractures in Adults. Am Fam Physician 2003;67:537-42.

18. Nevitt M. The Association of radiographycally detected vertebral fractures with back pain. Ann Int Med 1998;128:793

19. Green A. Does this woman have osteoporosis. JAMA 2004; 292:2890

20. Eastell R. Treatment of postmenopausal osteoporosis. N Engl J Med 1998;338:736

21. Rossen C. Postmenopausal Osteoporosis. N Engl J Med 2005; 353:595-603.

22. Delmas P. Treatment of Postmenopausal Osteoporosis. Lancet 2002;359:2018

23. Melton M. Evidence of two syndromes of involution osteoporosis. Am J Med 1983; 75:899-901.

24. Ensrud K. Low fractional calcium absorption increases the risk for hip fracture Ann Int Med 2000; 132:345

25. Cummings S. Epidemiology of Osteoporotic fractures. Lancet 2002; 359:1761-8.

26. Weinstein R. Glucocorticoid induced bone disease. N Engl J Med 2011; 365:62

27. Adler R., Hochberg MC. Suggested guidelines for evaluation and treatment of Glucocorticoid induced osteoporosis. Arch Int Med 2003; 163:2619-2624.

28. Kumar R. Glucocorticoid induced Osteoporosis. Curr Opinion Hypertens Nephrol 2001; 10:589595.

29. Buckley LM, Leib ES, Cartularo KS, Vacek PM, Cooper SM. Calcium and vitamin D3 supplementation prevents bone loss in the spine secondary to low-dose corticosteroids in patients with rheumatoid arthritis. A randomized, double-blind, placebo-controlled trial. Ann Intern Med 1996; 125:961-8.

30. Altkorn D, Cifu AS.Screening for Osteoporosis. JAMA 2015; 313:1467-1468.

31. Nelson H, Helfand M, Woolf SH, Allan JD. Screening for Postmenopausal Osteoporosis: a review of the evidence for the US Preventive Task Force. Ann Int Med 2002; 137:529-41.

32. Rud B, Jensen JE, Mosekilde L, Nielsen SP, Hilden J, Abrahamsen B. Performance of four clinical screening tools to select peri and early postmenopausal women for dual X-ray absorptiometry. Osteoporosis Int. 2005; 16:764-72.

33. Schurman L, Bagur A, Claus-Hermberg H, Messina OD. Guías 2012 para el diagnóstico, la prevención y el tratamiento de la osteoporosis. MEDICINA (Buenos Aires) 2013; 73:55-74,

34. Mauck K Use of clinical prediction rules in detecting osteoporosis in a population. Arch Int Med. 2005; 165:530

35. Cummings SR, Cawthon PM, Ensrud KE, et al. Osteoporotic Fractures in Men (MrOS) Research Groups. Dand risk of hip and nonvertebral fractures in older men: a prospective study and comparison with older women. J Bone Miner Res. 2006; 21:1550-6

36. Ensrud K. A comparison of prediction models for fractures in older patients Arch Int Med 2009; 169: 2087 
37. Poole K. Bisphosphonates in the treatment of Osteoporosis BMJ 2012; 344:e3211 doi: 10.1136/bmj.e3211

38. Spyridoula M. Biphosphonates for the prevention and treatment of osteoporosis. BMJ 2015; 351: h3783

39. Sarli M, Fradinger E, Morillo S. Tratamiento de la osteoporosis postmenopáusica con pamidronato intravenoso en pacientes con patología esofagogástrica. MEDICINA 1998; 58: 446-52.

40. Viiss $M$. The effect of intravenous pamidronate versus oral alendronate on bone mineral density in patients with osteoporosis. Osteoporosis Int 2005; 16:1432

41. Ekeman W. Treatment with intravenous pamidronate is a good alternative in case of gastrointestinal side effects or contraindications for oral bisphosphonates. BMC 2009;10:86. doi: 10.1186/1471-2474-10-86

42. South J. Osteoporosis nonpharmacologic and pharmacologic treatment. Am Fam Phys 2001; 83:1121

43. Michaëlsson K. Calcium supplements do not prevent fractures. BMJ 2015; 351:h4825 doi: 10.1136/bmj.h4825.

44. Bauer D. Calcium suplements and fracture prevention. N Engl J Med 2013; 369:1537

45. Bischoff A. Vitamin D. A meta-analysis of randomized controlled trials. JAMA 2005; 293:2257

46. Chung M, Lee J. Vitamin D With or Without Calcium Supplementation for Prevention of Cancer and Fractures: An Updated Meta-analysis for the U.S. Preventive Services Task Force Ann Intern Med. 2011;155:827-838.

47. Bischoff-Ferrari HA, Dawson-Hughes B, Willett WC, et al. Effect of Vitamin D on falls: a metaanalysis. JAMA 2004; 291:1999-2006.

48. Bolland MJ. Calcium intake and risk of fracture: systematic review. BMJ 2015;351: h4580 doi: 10.1136/bmj.h4580

49. Bolland MJ. Avenell A, Baron JA, et al. Effect of calcium supplements on risk of myocardial infarction and cardiovascular events: meta-analysis. BMJ 2010; 341:c3691 doi:10.1136/bmj.c3691

50. Bolland M. Calcium and cardiovascular risk. Australian prescriber 2013; 36:5-8.

51. Wang L, Manson JE, Song Y, Sesso HD. Systematic review: vitamin D and calcium supplementation in prevention of cardiovascular events. Ann Intern Med 2010; 152:315-23.

52. Black D. Randomized trial of alendronate effect on risk of fracture in postmenopausal women. Lancet 1996;348:1535-1541.

53. Cummings S. Effect of Alendronate on risk of Fracture in women with low bone density. JAMA 1998; 280:24/2077-83.

54. Harris S. Effects of Risedronate on vertebral and no vertebral fractures. JAMA 1999; 282:134452.

55. Mc Clung. Effect of Risedronate on he risk of Hip fracture N Engl J.Med. 2001; 344:5/333-41

56. Chesnut IC, Skag A, Christiansen C, Recker R, Stakkestad JA, Hoiseth A, et al. Effects of oral ibandronate administered daily or intermittently on fracture risk in postmenopausal osteoporosis. J Bone Miner Res. 2004;19:1241-9.

57. Hosking D. Prevention of bone loss with alendronate N. Engl. J. Med. 1998; 338:485-491.

58. Rizzoli R, for The Alendronate Once-Weekly Study Group. Two-year results of once-weekly administration of alendronate $70 \mathrm{mg}$ for the treatment of postmenopausal osteoporosis. J Bone Miner Res. 2002; 17:1988-1996.

59. Brown JP, Kendler DL, McClung MR, et al. The efficacy and tolerability of risedronate once a week for the treatment of postmenopausal osteoporosis. Calcif Tissue Int. 2002; 71:103-111.

60. Reginster JY, Adami S, Lakatos P, et al. Efficacy and tolerability of once-monthly oral ibandronate in postmenopausal osteoporosis: 2 year results from the MOBILE study. Ann Rheum Dis. 2006; 65:654-661.

61. Wells GA, Cranney A, Peterson J, et al. Alendronate for the primary and secondary prevention of osteoporotic fractures in postmenopausal women. Cochrane Database Syst Rev. 2008; 23(1):CD001155.

62. Effects of Continuing or Stopping Alendronate After 5 Years of Treatment The Fracture Intervention Trial Long-term Extension (FLEX): A Randomized Trial. JAMA. 2006; 296:29272938.

63. Cathleen S. Colon-Emeric. Ten vs Five Years of Bisphosphonate Treatment for Postmenopausal Osteoporosis Enough of a Good Thing. JAMA 2006; 296: 2968

64. Whitaker M. Bisphosphonates for Osteoporosis - Where Do We Go from Here? NEJM. 10.1056/nejmp1202619 nejm.org 
65. Abrhamsen Bo. Bisphosphonates adverse effects. Curr Op Rheumatol 2010; 22:1470.

66. Recker R. Safety of Bisphosphonates in the Treatment of Osteoporosis. Am J Med 2009; 122: 22.

67. Green J. Oral bisphosphonates and risk of cancer of oesophagus, stomach, and colorectum: case-control analysis within a UK primary care cohort Cite this as. BMJ 2010; 341:c4444

68. Vinogradova $Y$. Exposure to bisphosphonates and risk of gastrointestinal cancers: series of nested case-control studies with Q Research and CPRD data BMJ 2013; 356:f114 doi: 10.1136/bmj.f114

69. Davidson K, et al. Assessing fracture risk and effects of osteoporosis drugs. Am J Med 2009; 122: 992.

70. Black DM, Schwartz AV, Ensrud KE, et al. Effects of Continuing or Stopping Alendronate After 5 Years of Treatment. The Fracture Intervention Trial Long-term Extension (FLEX): A Randomized Trial. JAMA. 2006; 296:2927-2938.

71. Schwartz AV, Bauer DC, Cummings SR. Efficacy of Continued Alendronate for Fractures in Women With and Without Prevalent Vertebral Fracture: The FLEX Trial. J Bone Miner Res 2010; 25:976-982.

72. Chan SS. Subtrochanteric Femoral Fractures in Patients Receiving Long-Term Alendronate Therapy: Imaging Features. AJR 2010; 194:1581-1586.

73. Briesacher BA. Adoption of Once-monthly Oral Bisphosphonates and the Impact on Adherence. Am J Med 2010; 123:275-280.

74. Eekman DA, Vis M. Treatment with intravenous pamidronate is a good alternative in case of gastrointestinal side effects or contraindications for oral bisphosphonates. BMC Musculoskeletal Disord 2009; 10: 86 doi:10.1186/1471-2474-10-86

75. Black DM, Delmas PD. Once-Yearly Zoledronic Acid for Treatment of Postmenopausal Osteoporosis. N Engl J Med 2007; 356:1809-22.

76. Eisman JA, Civitelli R, Adami S, et al. Efficacy and tolerability of intravenous ibandronate injections in postmenopausal osteoporosis: 2-year results from the DIVA study. J Rheumatol. 2008; 35:488-97.

77. Garjón Parra J. Ibandronato y prevención de fracturas no vertebrales. Lectura crítica de dos metanálisis de datos individuales de pacientes. BIT 2010; 18 (1)

78. Delmas PD, Adami S, Strugala C, et al. Intravenous ibandronate injections in postmenopausal women with osteoporosis: one-year results from the Dosing Intravenous Administration Study. Arthritis Rheum.2006; 54:1838-1846. 